\title{
Revealing the Microscopic Real-Space Excursion of a Laser-Driven Electron
}

\author{
Heiko G. Kurz, ${ }^{1,2,}$ Martin Kretschmar, ${ }^{1,2}$ Thomas Binhammer, ${ }^{3}$ Tamas Nagy, ${ }^{1,4}$ Detlev Ristau, ${ }^{1,5}$ \\ Manfred Lein, ${ }^{2,6}$ Uwe Morgner, ${ }^{1,2,5}$ and Milutin Kovačev ${ }^{1,2}$ \\ ${ }^{1}$ Leibniz. Universität Hannover, Institut für Quantenoptik, Welfengarten 1, D-30167 Hannover, Germany \\ ${ }^{2}$ QUEST, Centre for Quantum Engineering and Space-Time Research, \\ Welfengarten 1, D-30167 Hannover, Germany \\ ${ }^{3}$ VENTEON GmbH, Hollerithallee 17, D-30419 Hannover, Germany \\ ${ }^{4}$ Laser Laboratorium Göttingen e.V., Hans-Adolf-Krebs-Weg 1, D-37077 Göttingen, Germany \\ ${ }^{5}$ Laser Zentrum Hannover e.V., Hollerithallee 8, D-30419 Hannover, Germany \\ ${ }^{6}$ Leibniz, Universität Hannover, Institut für Theoretische Physik, \\ Appelstraße 2, D-30167 Hannover, Germany
}

(Received 15 January 2016; published 24 August 2016)

\begin{abstract}
High-order harmonic spectroscopy allows one to extract information on fundamental quantum processes, such as the exit time in the tunneling of an electron through a barrier with attosecond time resolution and molecular structure with angstrom spatial resolution. Here, we study the spatial motion of the electron during high-order harmonic generation in an in situ pump-probe measurement using highdensity liquid water droplets as a target. We show that molecules adjacent to the emitting electron-ion pair can disrupt the electron's trajectory when positioned within the range of the maximum electronic excursion distance. This allows us to use the parent ion and the neighboring molecules as boundaries for the electronic motion to measure the maximum electronic excursion distance during the high-order harmonic generation process. Our analysis of the process is relevant for optimizing high-harmonic yields in dense media.
\end{abstract}

DOI: 10.1103/PhysRevX.6.031029

\section{INTRODUCTION}

Light-matter interaction in the strong-field regime discloses a broad range of quantum phenomena [1]. When the electric field strength of the incident light reaches the magnitude of the atomic or molecular Coulomb field, the potential is distorted and an electron may tunnel through the resulting barrier [2,3]. The released electron is accelerated upon tunnel ionization and can recombine with its parent ion by emitting a harmonic photon. This extremely nonlinear process is known as high-order harmonic generation (HHG) [4-7], and allows us to extract information on fundamental quantum processes, such as the tunneling of an electron through a barrier [8] or molecular properties [9-13] with attosecond time resolution [14] and angstrom spatial resolution $[15,16]$. The temporal characterization of the HHG process has been studied intensively, in particular, concerning the ionization and recombination times [8,17-22]. While the tunnel ionization and the recombination are quantum-mechanical effects that result in deviations from a semiclassical description of HHG [8],

\footnotetext{
* Corresponding author. kurz@iqo.uni-hannover.de

Published by the American Physical Society under the terms of the Creative Commons Attribution 3.0 License. Further distribution of this work must maintain attribution to the author(s) and the published article's title, journal citation, and DOI.
}

Subject Areas: Atomic and Molecular Physics, Optics, Plasma Physics the electronic excursion in the continuum can still be treated semiclassically by assigning a different classical trajectory to every time of ionization [4], leading to similar results as quantum trajectories $[17,18,23,24]$ for the maximum electron excursion distance from the parent ion. However, the spatial propagation of the electron in real space and possible perturbations to the electronic trajectory were only supposed $[25,26]$ and are still little explored. This is especially of interest regarding recent experiments in high-density targets with long driver wavelengths [27] where the electron may be scattered on its excursion by an adjacent atom, which is a previously unnoticed effect. High-harmonic spectroscopical measurements of the dissociation process in molecules indicate indeed that the amplitude of the emitted radiation is highly sensitive to atoms close to the parent ion [28].

We apply a high-density target to place a molecule within the electron's trajectory, which can induce a severe distortion to HHG, resulting even in the extinction of the emission process. This approach enables us to use the HHGgenerating medium itself for the measurement of the maximum electronic displacement during HHG, where the parent ion serves as a lower limit while the neighboring molecule serves as a soft upper limit. We reveal the spatial electronic excursion and are able to confirm the legitimacy of the semiclassical treatment of the electron on its excursion by comparing the extreme-ultraviolet (XUV) radiation emitted during this process with the calculated trajectories. 


\section{MICROSCOPIC DYNAMICS IN THE MEDIUM}

In order to determine the relevant distance between the parent ion and the adjacent molecule for inducing a perturbation, the electronic motion is calculated in the semiclassical picture. Newton's equation of motion is solved for an electron driven by the force of the laser field. As a result, the ionization time $t_{i}$, the recombination time $t_{r}$, and the return energy of the electron $E_{r}$ are obtained for every photon energy of the harmonic radiation, which allows a temporal characterization of the electron dynamics during the HHG process [4]. The trajectories leading to the emission of harmonic radiation are depicted in Fig. 1(a). The length of the trajectories depends on $t_{i}$, and one can distinguish the trajectories by the time spent within the continuum into the cutoff, the short and the long trajectory. The cutoff trajectory results in the largest energy release of $E_{\text {cutoff }}=I_{p}+3.17 U_{p}$ [4], where $I_{p}$ is the ionization potential of the target atom and $U_{p}$ is the ponderomotive potential of the driving field. The short and the long trajectory emit equal photon energies. However, they vary in their maximum excursion from the parent ion $x_{\max }$, which is on the angstrom level.

The difference in $x_{\max }$ gives the opportunity to follow the electronic movement, and to selectively probe the trajectories by reducing the distance between the particles in the target medium and thus inducing a possibility for a perturbation. Hence, we can use the molecule in the path of the electron to suppress the recombination step with the generating medium itself and measure $x_{\max }$ by observing the decrease of the emitted radiation. The long trajectories have a larger value of $x_{\max }$ and are therefore perturbed earlier as the distance between the ion and the adjacent molecule is reduced, subsequently followed by the cutoff and the short trajectories. The maximum electronic excursion depends on two parameters: the trajectory and the intensity of the driving field $I$, which we discuss in the following.

For a given harmonic order, we may consider generation at the position in the laser intensity profile, where this harmonic order is generated via the cutoff harmonic. The application of the cutoff law [4] enables us to express $x_{\max }$ as a function of the harmonic order. As indicated by the solid blue line in Fig. 1(b), the maximum displacement for HHG via the cutoff trajectory increases with the harmonic order. When a given harmonic order is generated at an intensity above its cutoff intensity, then this harmonic order is situated within the plateau region; i.e., it is generated via short and long trajectories [5]. As a consequence, $x_{\max }$ is increased for long trajectories while it decreases for short trajectories as a function of intensity (see Supplemental Material [29]). The color scale in Fig. 1(b) indicates the change of $x_{\max }$ for the short trajectory contributing to a specific harmonic order. From here on we concentrate on the short trajectories, since we select them in our experiment due to its lower value of $x_{\max }$. An increase in the intensity of the driving field (from blue to white) equals a change of the generating quantum path (from the cutoff into the plateau), reducing $x_{\max }$. For example, an increase of the peak intensity from $I=1.1 \times 10^{14} \mathrm{~W} / \mathrm{cm}^{2}$ to $I=$ $2.2 \times 10^{14} \mathrm{~W} / \mathrm{cm}^{2}$ leads to a reduction of $x_{\max }$ from $1.04 \mathrm{~nm}$ (cutoff trajectory, dashed black line) to $0.65 \mathrm{~nm}$

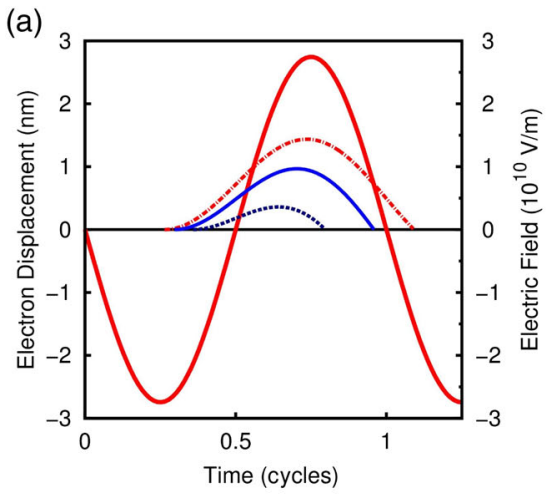

(b)

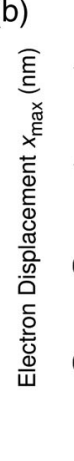

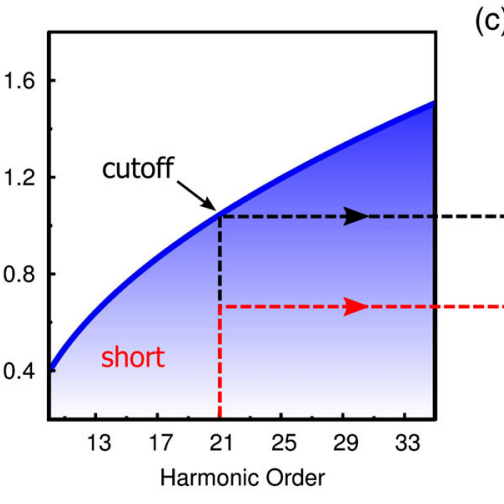

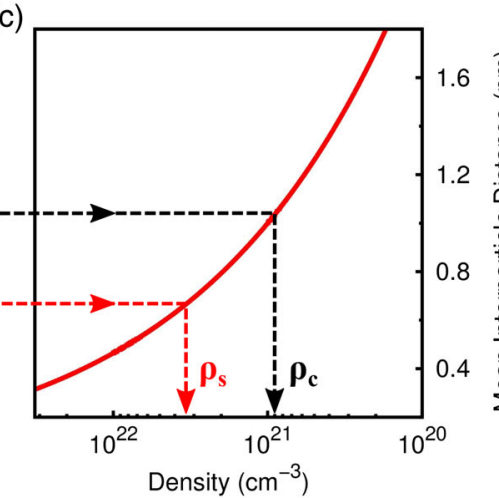

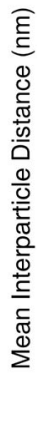

FIG. 1. Determination of the maximum tolerable target density for HHG. (a) Calculated electron trajectories during HHG. Depending on the time of ionization by the electric field (solid red line), different quantum paths contribute to the HHG process, such as the short (dotted blue line), the cutoff (solid blue line), and the long trajectory (dashed red line). Different trajectories may result in the same photon energy, but can be distinguished by the time spent within the continuum and by the maximum displacement of the electron $x_{\max }$. (b) Calculated range of maximum electron displacement as a function of harmonic order. The blue solid line indicates the displacement along the cutoff trajectory. As the driving intensity increases, the maximum excursion of the electron contributing to a fixed harmonic order decreases (color scale) since the harmonic shifts from the cutoff into the plateau region, and the process occurs via the short quantum path. (c) Mean interparticle distance versus target density (solid red line). Comparing (b) and (c) allows us to determine the maximum tolerable density of the target, upon which a perturbation of electronic trajectories sets in and the HHG process is suppressed. Depending on the trajectory, two values are obtained: $\rho_{c}$ (dashed black arrow exemplarily for the 21 st order) for the cutoff, and a lower, intensity-dependent value for the short trajectory $\rho_{s}$ (dashed red arrow). 


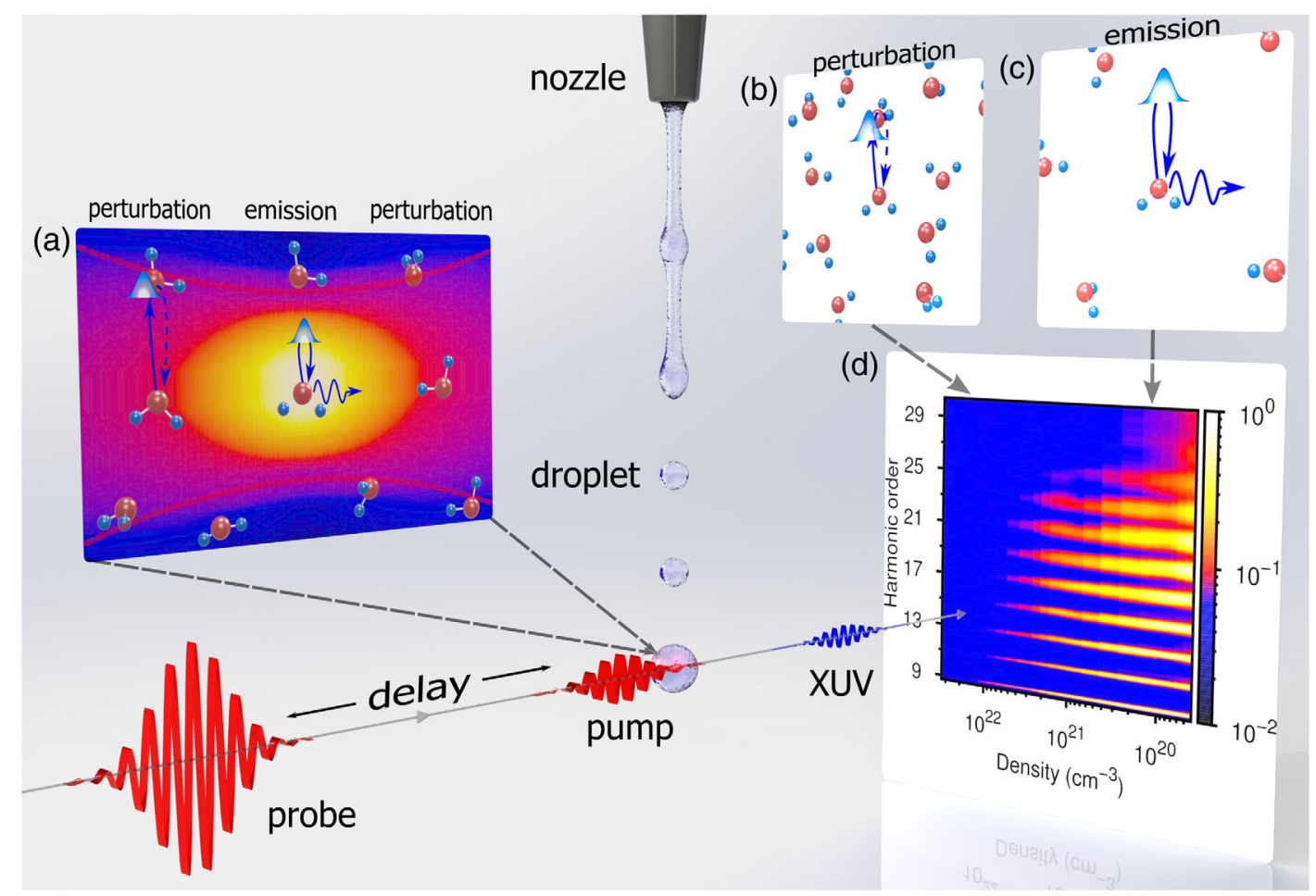

FIG. 2. Schematic illustration of the experimental setup. Two intense, time-delayed laser pulses interact with a liquid droplet in the vacuum. While the first pulse (pump) makes the droplet expand via laser-induced optical breakdown, the second pulse (probe) generates harmonic radiation. Depending on the time delay between the pulses, different densities of the target are accessible for HHG. (a) Different intensities lead to different electronic excursions, such that the electron can be perturbed by surrounding molecules. (b),(c) For a fixed intensity of the probe pulse, this gives access to probe the electronic excursion by increasing the target's density and detecting the harmonic yield. At high densities, the mean interparticle distance of the target's components matches the electronic excursion during HHG, which allows us to induce a perturbation to the quantum paths and (d) results in a decreasing harmonic signal as a function of the density.

(short trajectory, dashed red line) for the 21 st harmonic order. Thus, we can control the maximum displacement of the electron contributing to a specific harmonic order by tuning the intensity of the driving field. With this approach, we obtain two values for $x_{\max }$ for each harmonic order, which define an intensity-dependent range of the displacement for each harmonic order: the first one for HHG via the cutoff trajectory, which is an upper limit, and a second value corresponding to the actual laser peak intensity.

As we show above, the range of $x_{\max }$ is within the angstrom scale. In order to induce a perturbation to the electronic trajectories, the distance between two particles in the target must be comparable to $x_{\max }$. HHG, however, is performed in a macroscopic ensemble with many particles and differing distances. We therefore consider the mean interparticle distance $\bar{r}=\sqrt[3]{1 / \rho}$ to account for the macroscopic aspect [30] and to obtain an experimentally accessible measure for the onset of the perturbation: the number density $\rho$ as depicted in Fig. 1(c). A comparison between $x_{\max }$ and $\bar{r}$ enables us to map the maximum displacement onto a maximum tolerable target density. Two density limits are obtained for each harmonic order, the first for the cutoff trajectory $\rho_{c}$ (dashed black line in Fig. 1 exemplarily for the 21st harmonic order) and the second, intensitydependent value for the short trajectory $\rho_{s}$ (dashed red line). Within the target, however, the distance between the molecules deviates from the average value $\bar{r}$. We therefore take the statistical probability into account that the electron on its trajectory encounters a water molecule, which can be formulated when information on the electron-molecule scattering cross section is available. The probability $p$ for an electron to remain undisturbed along its trajectory is

$$
\begin{aligned}
p & \propto \exp \left(-\rho \alpha_{T}\left(2 U_{p}\right)^{-0.1} \sqrt{\frac{2}{m_{e}}} \frac{1}{\omega_{L}} 2.75\left|\tau_{i}\right|^{0.65}\right) \\
& =\exp \left(-\rho V_{\text {scat }}\right),
\end{aligned}
$$

where $U_{p}$ is the ponderomotive potential, $\alpha_{T}\left(2 U_{p}\right)^{-0.1}$ considers the energy-dependent electron-molecule cross section [31], $m_{e}$ is the mass of the electron, $\omega_{L}$ is the frequency of the driving field, and $\tau_{i}=t_{i} \omega_{L}$ is a measure for the ionization time $t_{i}$ in a time-dependent electric field of the form $E(t)=E_{0} \sin \left(\omega_{L} t\right)$, where $t_{i}$ must be chosen in the quarter cycle $\left[-\pi /\left(2 \omega_{L}\right), 0\right]$ (cf. Supplemental Material [29] for details). Equation (1) depends on $\rho$ and the 
scattering volume $V_{\text {scat }}$ of the electron, which is for the short trajectory within the order of $V_{\text {scat }} \approx 10^{-22} \mathrm{~cm}^{3}$. We implement the probability $p$ into a macroscopic model for the calculation of the signal $S_{q}$ of the $q$ th harmonic order [32,33], where phase-matching aspects are considered by the coherence length. This allows us to study the coherent XUV buildup as a function of the target density beyond pure phase matching.

\section{EXPERIMENTAL CONFIRMATION OF THE MODEL}

In order to experimentally satisfy the requirements of a high-density target with a spatial confinement, we use a liquid water-droplet setup under vacuum conditions as a target for HHG [34] (cf. Fig. 2). For generating harmonic radiation from targets with tunable density, we focus intense laser pulses with a central wavelength of $780 \mathrm{~nm}$ and a pulse duration of $35 \mathrm{fs}$ in a pump-probe setup onto the $15-\mu \mathrm{m}$-sized water droplets. The pump pulse therein makes the droplet expand through a laser-induced optical breakdown [35] in a way that the target's composition is dominated by neutral water molecules with a total ionization below 10\% (cf. Supplemental Material [29]). The pump pulse does not contribute to HHG due to the high density of the target, meaning that the harmonic radiation is solely generated by the probe pulse. We select the short trajectory by phase matching via the focal position [36] and measure the harmonic spectrum as a function of the pumpprobe delay $t$. In order to determine the density of the target, we apply a model from plasma physics to calculate the density as a function of $t$ [34]. The model allows us to map $t$ onto $\rho$ (see Supplemental Material [29]), such that we obtain access to the harmonic yield as a function of the density of the target, reaching from $3.35 \times 10^{22} \mathrm{~cm}^{-3}$ to $1 \times 10^{17} \mathrm{~cm}^{-3}$ [cf. Fig. 2(d)].

Since the densities available with the liquid droplet are sufficiently high to provide mean interparticle distances matching the electronic excursion, we are able to experimentally test the model for the maximum tolerable density. Figure 3(a) depicts the normalized experimental spectra of the harmonic radiation for different densities. The water droplet is expanded with an intensity of the pump pulse of $I_{\text {pump }}=4.5 \times 10^{14} \mathrm{~W} / \mathrm{cm}^{2}$. In order to generate the presented harmonic orders via the short trajectory, the intensity of the probe pulse has been set to $I_{\text {probe }}=$ $1.4 \times 10^{15} \mathrm{~W} / \mathrm{cm}^{2}$. At high densities, no harmonic radiation is emitted, but the signal increases as the density is lowered and higher orders successively appear. A more detailed study is given in Fig. 3(b), which depicts an outline of the density-mapped signal of the 19th harmonic order for a larger density range. It is compared with two simulations, the first excluding (gray line) and the second including (blue line) the possibility of electron-molecule scattering. At low densities, the probability for a perturbation of
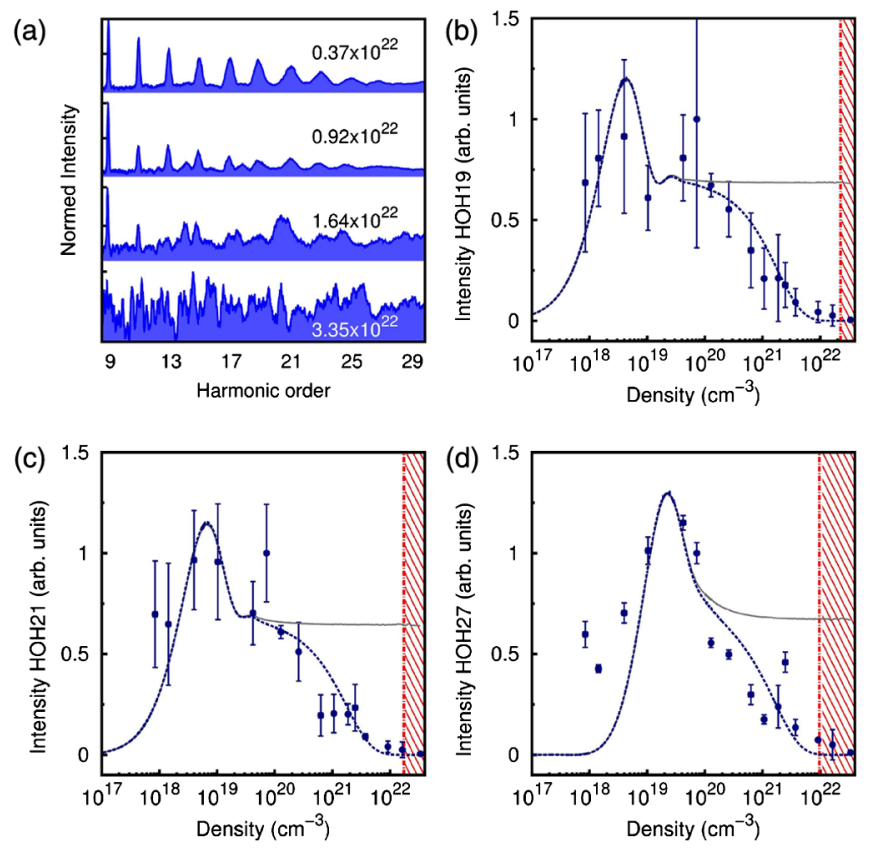

FIG. 3. Observing the perturbation of electronic trajectories. (a) Spectra of the harmonic radiation for different densities of the target. (b)-(d) Density-mapped harmonic radiation of the 19th, 21 st, and 27th order, respectively (blue dots). The signal increases for low densities. A loss in the signal of the harmonic radiation is observed for increasing density due to a higher probability of a perturbation of the electronic trajectory by an adjacent molecule. The measured data are compared with a calculation of the harmonic signal as a function of the density for two cases: including (blue line) and excluding (gray line) a perturbation of electronic trajectories. All trajectories are perturbed for densities $\rho_{s}$ corresponding to the excursion distance of the short trajectory, and no harmonic radiation is emitted (red area). Since $x_{\max }$ increases as a function of the harmonic order, $\rho_{s}$ shifts towards lower values with increasing harmonic order.

electronic trajectories is small. Thus, the signal increases as a function of the density. After reaching a maximum, a decrease is observed until a density of $\rho \approx 2 \times 10^{19} \mathrm{~cm}^{-3}$ is reached. This decrease can be attributed to the onset of reabsorption of the XUV radiation. For higher values of $\rho$, the calculation without scattering of trajectories (gray line) shows an almost constant, slowly decreasing signal, whereas the simulation including the trajectory perturbation (blue line), in agreement with the experimental data (blue dots), shows a coinciding fast decrease. This discrepancy clearly demonstrates the influence of neighboring molecules on an electron moving in the continuum. When the perturbation is excluded (gray line), the almost constant signal can be traced back to an increase of the reabsorption at higher density, which is compensated by a higher number of emitters (see Supplemental Material [29]). If the perturbation is included (blue line), the distortion of electronic trajectories becomes more probable with increasing density [cf. Eq. (1)], which leads to a decreasing signal. The 
same observation is made for higher orders, as depicted in Figs. 3(c) and 3(d) for the 21st and the 27th order, respectively. The model accurately describes the observations made in the experiments. For benchmarking the simulation, we apply our model to recent findings of HHG in a high-density helium target with mid-IR driver pulses [27,37]. Our calculations for the latter case indicate that the distortion of electronic trajectories by other $\mathrm{He}$ atoms is of minor influence because the total cross section for electron-helium collisions is smaller than the electron-water cross section (cf. Supplemental Material for details [29]).

The HHG process breaks down for densities $\rho>\rho_{s}$ [red area in Figs. 3(b)-3(d)], which is the density limit derived in Fig. 1. The disappearance of the harmonic radiation is confirmed by the comparison with the spectra in Fig. 3(a), where the harmonic peak structure is lost for densities $\rho>\rho_{s}$. Because of the increase of the electronic displacement as a function of the harmonic order, the trajectories attributed to higher orders are perturbed at a lower density. This makes higher orders the ideal instrument to probe the maximum electronic displacement, as shown in the case of the 27th harmonic order in Fig. 3(d). The measurements confirm the model for the perturbation of electronic trajectories, and we can use the neighboring molecules to confine the emission process and to measure the maximum electronic displacement $x_{\max }$.

\section{STEERING ELECTRONIC TRAJECTORIES}

Furthermore, we are able to directly manipulate the electronic excursion with the intensity of the driving field. Since the displacement of the electron contributing to a certain harmonic order becomes smaller as we increase $I_{\text {probe }}$, we can select a fixed target density of $\rho=\rho_{s}$ and expect a change of the emission process from incoherent to coherent emission of radiation as $I_{\text {probe }}$ increases. To this end, Fig. 4(a) shows the spectra of harmonic radiation as a function of the intensity of the probe pulse at a density of $\rho=2.5 \times 10^{21} \mathrm{~cm}^{-3}$. At an intensity of $I_{\text {probe }}=0.2 \times 10^{15} \mathrm{~W} / \mathrm{cm}^{2}$, harmonic radiation is detected up to the 21st order, although $I_{\text {probe }}$ is sufficiently high to generate higher orders. However, the target is dense enough to disable HHG for the higher orders (red area), which we can attribute to the perturbation of the short trajectories. The absence of higher orders also allows us to exclude contributions from lower orbitals of the water molecule, such as HOMO-1 and HOMO-2 [38,39], since their higher ionization potential shifts $x_{\max }$ towards lower values and, therefore, would allow HHG in the red area. As we increase $I_{\text {probe }}$, the maximum electronic displacement is reduced, such that higher orders up to the 29th order consecutively appear.

Applying the semiclassical model, the change of the intensity from $0.2 \times 10^{15} \mathrm{~W} / \mathrm{cm}^{2}$ to $0.4 \times 10^{15} \mathrm{~W} / \mathrm{cm}^{2}$ would correspond to a change of $\Delta x_{\max } \approx 1.8 \AA$ in the case of the 25 th order, and of $2.4 \AA$ for the 27 th order. Following the attosecond electronic dynamics by tuning the intensity of the driving field is therefore possible, and we are able to probe $x_{\max }$ via $I_{\text {probe }}$ or by controlling $\rho$. Hence, we combine both parameters in Fig. 4(b) to get a full picture of the observation and control of the electronic excursion. We measure the signal of the 29th harmonic spectral line since it has the largest value of $x_{\max }$. As already observed in Fig. 3 (red area), the signal vanishes for $\rho>\rho_{s}$ (dashed red line) and at intensities below $0.4 \times 10^{15} \mathrm{~W} / \mathrm{cm}^{2}$. We can switch on the corresponding trajectories either by increasing the driving intensity or by decreasing the target density. (a)

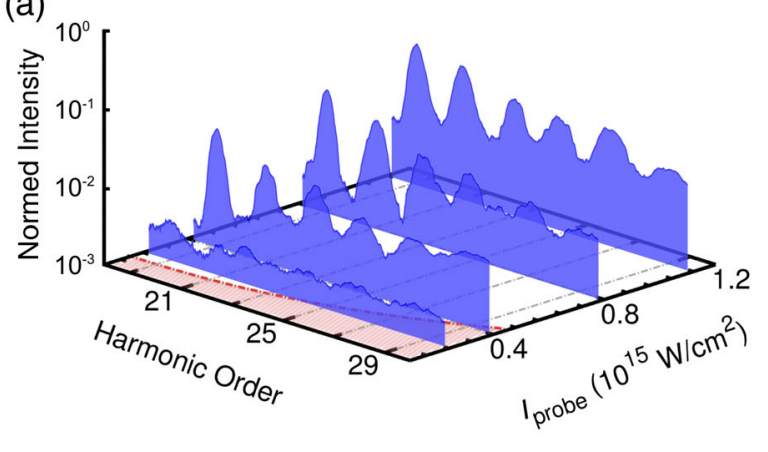

(b)

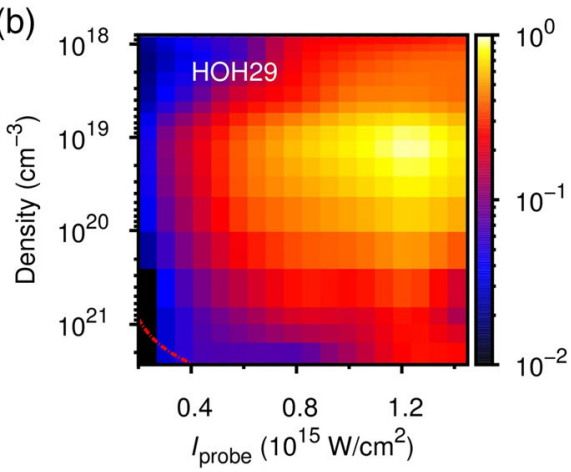

FIG. 4. Controlling the electronic excursion with the driving intensity. (a) Spectra of harmonic radiation as a function of the intensity of the probe pulse for a fixed density of the target. No harmonic radiation is observed within the red area, where the electronic displacement is larger than the mean interparticle distance. The displacement is reduced with increasing intensity, and the emission process turns from incoherent to coherent. (b) Signal of the 29th harmonic order as a function of the target density and the intensity of the driving pulse. A perturbation of the trajectories becomes more probable for densities higher than the position of the maximum in intensity. Again, no harmonic radiation is detected for densities and intensities where the electronic excursion matches the mean interparticle distance assigned to the short trajectory $\left(\rho>\rho_{s}\right.$, dashed red line). 


\section{CONCLUSION AND OUTLOOK}

The presented method enables the spatial observation of attosecond electron dynamics. By introducing a perturbation to the electronic trajectories during HHG with adjacent molecules, we can probe the displacement and verify the semiclassical treatment of the electron during its excursion [4]. Moreover, we are able to switch trajectories on and off, which allows us to observe subcycle dynamics during the light-matter interaction and opens a possible route to control the intrinsic attochirp of HHG [18]. Apart from phase-matching-determined HHG, we find a high-density regime that is relevant for optimizing the HHG process [27,33]. High-density targets are especially interesting as they feature new possibilities for attosecond physics $[13,40-47]$. The droplet offers the possibility to study attosecond dynamics [48] of different microscopic states, such as atoms, molecules, and clusters [49-51] in a single experiment with surpassing accuracy. Our method has the potential to fully characterize the HHG process in a single setup, when combined with a measurement of $t_{i}, t_{r}$, and $E_{r}$ in a two-color, in situ experiment [8,19], and may even open a new route to the generation of isolated attosecond pulses by confining the emission process to a single event by the medium itself.

\section{ACKNOWLEDGMENTS}

We thank Ahmed Msellati for assistance with the visualization of the setup. We are grateful for financial support from DFG project No. KO 3798/1-1 and the Air Force Office of Scientific Research under Grant No. FA9550-12-1-0482. This research was supported by the cluster of excellence QUEST-Centre for Quantum Engineering and Space-Time Research.

[1] F. Krausz and M. Ivanov, Attosecond Physics, Rev. Mod. Phys. 81, 163 (2009).

[2] L. Keldysh, Quantum Transport Equations for High Electric Fields, Sov. Phys. JETP 20, 1307 (1965).

[3] M. Uiberacker et al., Attosecond Real-Time Observation of Electron Tunnelling in Atoms, Nature (London) 446, 627 (2007).

[4] P. Corkum, Plasma Perspective on Strong Field Multiphoton Ionization, Phys. Rev. Lett. 71, 1994 (1993).

[5] M. Lewenstein, Ph. Balcou, M. Y. Ivanov, A. L'Huillier, and P. B. Corkum, Theory of High Harmonic Generation by Low-Frequency Laser Fields, Phys. Rev. A 49, 2117 (1994).

[6] A. McPherson, G. Gibson, H. Jara, U. Johann, T. S. Luk, I. A. McIntyre, K. Boyer, and C. K. Rhodes, Studies of Multphoton Production of Vacuum Ultraviolet-Radiation in the Rare-Gases, J. Opt. Soc. Am. B 4, 595 (1987).

[7] M. Ferray, A L'Huillier, X. F. Li, L. A. Lompre, G. Mainfray, and C. Manus, Multiple-Harmonic Conversion of 1064-nm Radiation in Rare-Gases, J. Phys. B 21, L31 (1988).
[8] D. Shafir, H. Soifer, B. D. Bruner, M. Dagan, Y. Mairesse, S. Patchkovskii, M. Y. Ivanov, O. Smirnova, and N. Dudovich, Resolving the Time When an Electron Exits a Tunnelling Barrier, Nature (London) 485, 343 (2012).

[9] R. Torres et al., Probing Orbital Structure of Polyatomic Molecules by High-Order Harmonic Generation, Phys. Rev. Lett. 98, 203007 (2007).

[10] B. K. McFarland, J. Farrell, P. H. Bucksbaum, and M. Gühr, High Harmonic Generation from Multiple Orbitals in $\mathrm{N}_{2}$, Science 322, 1232 (2008).

[11] W. Li, X. Zhou, R. Lock, S. Patchkovskii, A. Stolow, H. C. Kapteyn, and M. M. Murnane, Time-Resolved Dynamics in $\mathrm{N}_{2} \mathrm{O}_{4}$ Probed Using High Harmonic Generation, Science 322, 1207 (2008).

[12] O. Smirnova, Y. Mairesse, S. Patchkovskii, N. Dudovich, D. Villeneuve, P. Corkum, and M. Y. Ivanov, High Harmonic Interferometry of Multi-Electron Dynamics in Molecules, Nature (London) 460, 972 (2009).

[13] S. Haessler, V. Strelkov, L. B. Elouga Bom, M. Khokhlova, O. Gobert, J.-F. Hergott, F. Lepetit, M. Perdrix, T. Ozaki, and P. Salières, Phase Distortions of Attosecond Pulses Produced by Resonance-Enhanced High Harmonic Generation, New J. Phys. 15, 013051 (2013).

[14] M. Hentschel, R. Kienberger, Ch. Spielmann, G. A. Reider, N. Milosevic, T. Brabec, P. Corkum, U. Heinzmann, M. Drescher, and F. Krausz, Attosecond Metrology, Nature (London) 414, 509 (2001).

[15] J. Itatani, J. Levesque, D. Zeidler, H. Niikura, H. Pépin, J. C. Kieffer, P. B. Corkum, and D. M. Villeneuve, Tomographic Imaging of Molecular Orbitals, Nature (London) 432, 867 (2004).

[16] C. Vozzi, M. Negro, F. Calegari, G. Sansone, M. Nisoli, S. De Silvestri, and S. Stagira, Generalized Molecular Orbital Tomography, Nat. Phys. 7, 822 (2011).

[17] P. Salières et al., Feynman's Path-Integral Approach for Intense-Laser-Atom Interactions, Science 292, 902 (2001).

[18] Y. Mairesse et al., Attosecond Synchronization of HighHarmonic Soft X-Rays, Science 302, 1540 (2003).

[19] N. Dudovich, O. Smirnova, J. Levesque, Y. Mairesse, M. Y. Ivanov, D. M. Villeneuve, and P. B. Corkum, Measuring and Controlling the Birth of Attosecond XUV Pulses, Nat. Phys. 2, 781 (2006).

[20] P. Eckle, M. Smolarski, P. Schlup, J. Biegert, A. Staudte, M. Schöffler, H. G. Muller, R. Dörner, and U. Keller, Attosecond Angular Streaking, Nat. Phys. 4, 565 (2008).

[21] A. N. Pfeiffer, C. Cirelli, M. Smolarski, D. Dimitrovski, M. Abu-samha, L. B. Madsen, and U. Keller, Attoclock Reveals Natural Coordinates of the Laser-Induced Tunneling Current Flow in Atoms, Nat. Phys. 8, 76 (2012).

[22] J. Zhao and M. Lein, Determination of Ionization and Tunneling Times in High-Order Harmonic Generation, Phys. Rev. Lett. 111, 043901 (2013).

[23] R. M. Lock, S. Ramakrishna, X. Zhou, H. C. Kapteyn, M. M. Murnane, and T. Seideman, Extracting Continuum Electron Dynamics from High Harmonic Emission from Molecules, Phys. Rev. Lett. 108, 133901 (2012).

[24] K. T. Kim, C. Zhang, A. D. Shiner, S. E. Kirkwood, E. Frumker, G. Gariepy, A. Naumov, D. M. Villeneuve, and P. B. Corkum, Manipulation of Quantum Paths for 
Space-Time Characterization of Attosecond Pulses, Nat. Phys. 9, 159 (2013).

[25] A. Flettner, T. Pfeifer, D. Walter, C. Winterfeldt, C. Spielmann, and G. Gerber, High-Harmonic Generation and Plasma Radiation from Water Microdroplets, Appl. Phys. B 77, 747 (2003).

[26] V. V. Strelkov, V. T. Platonenko, and A. Becker, HighHarmonic Generation in a Dense Medium, Phys. Rev. A 71, 053808 (2005).

[27] T. Popmintchev et al., Bright Coherent Ultrahigh Harmonics in the keV X-Ray Regime from Mid-Infrared Femtosecond Lasers, Science 336, 1287 (2012).

[28] H. Wörner, D. Bertrand, J. B. Kartashov, P. Corkum, and D. Villeneuve, Following a Chemical Reaction Using HighHarmonic Interferometry, Nature (London) 466, 604 (2010).

[29] See Supplemental Material at http://link.aps.org/ supplemental/10.1103/PhysRevX.6.031029 for details on the calculation of the electronic trajectories, the determination of the maximum electronic displacement, the mapping of the time delay onto the density evolution. Furthermore, a model is developed to compare simulations of the harmonic yield including and excluding a perturbation of electronic trajectories with the measured data. The model is also used to calculate the XUV-signal from helium at high pressures with different driver wavelengths.

[30] P. Hertz, Über den Gegenseitigen Durchschnittlichen Abstand von Punkten, die mit Bekannter Mittlerer Dichte im Raume Angeordnet Sind, Math. Ann. 67, 387 (1909).

[31] Y. Itikawa and M. Mason, Cross Sections for Electron Collisions with Water Molecules, J. Phys. Chem. Ref. Data 34, 1 (2005).

[32] E. Constant, D. Garzella, P. Breger, E. Mével, Ch. Dorrer, C. Le Blanc, F. Salin, and P. Agostini, Optimizing High Harmonic Generation in Absorbing Gases: Model and Experiment, Phys. Rev. Lett. 82, 1668 (1999).

[33] J. Rothhardt, S. Hädrich, S. Demmler, M. Krebs, S. Fritzsche, J. Limpert, and A. Tünnermann, Enhancing the Macroscopic Yield of Narrow-Band High-Order Harmonic Generation by Fano Resonances, Phys. Rev. Lett. 112, 233002 (2014).

[34] H. G. Kurz, D. S. Steingrube, D. Ristau, M. Lein, U. Morgner, and M. Kovačev, High-Order-Harmonic Generation from Dense Water Microdroplets, Phys. Rev. A 87, 063811 (2013).

[35] Q. Feng, J. V. Moloney, A. C. Newell, E. M. Wright, K. Cook, P. K. Kennedy, D. X. Hammer, B. A. Rockwell, and C. R. Thompson, Theory and Simulation on the Threshold of Water Breakdown Induced by Focused Ultrashort Laser Pulses, IEEE J. Quantum Electron. 33, 127 (1997).

[36] P. Salières, A. L'Huillier, and M. Lewenstein, Coherence Control of High-Order Harmonics, Phys. Rev. Lett. 74, 3776 (1995).

[37] M.-C. Chen, P. Arpin, T. Popmintchev, M. Gerrity, B. Zhang, M. Seaberg, D. Popmintchev, M. M. Murnane, and H.C. Kapteyn, Bright, Coherent, Ultrafast Soft X-Ray Harmonics Spanning the Water Window from a Tabletop Light Source, Phys. Rev. Lett. 105, 173901 (2010).

[38] J. P. Farrell, S. Petretti, J. Förster, B. K. McFarland, L. S. Spector, Y. V. Vanne, P. Decleva, P. H. Bucksbaum, A. Saenz, and M. Gühr, Strong Field Ionization to Multiple Electronic States in Water, Phys. Rev. Lett. 107, 083001 (2011).

[39] S. Petretti, A. Saenz, A. Castro, and P. Decleva, Water Molecules in Ultrashort Intense Laser Fields, Chem. Phys. 414, 45 (2013).

[40] T. Ditmire, E. T. Gumbrell, R. A. Smith, J. W. G. Tisch, D. D. Meyerhofer, and M.H. R. Hutchinson, Spatial Coherence Measurement of Soft X-Ray Radiation Produced by High Order Harmonic Generation, Phys. Rev. Lett. 77, 4756 (1996).

[41] S. Ghimire, A. D. DiChiara, E. Sistrunk, P. Agostini, L. F. DiMauro, and D. A. Reis, Observation of High-Order Harmonic Generation in a Bulk Crystal, Nat. Phys. 7, 138 (2011).

[42] J. A. Wheeler, A. Borot, S. Monchocé, H. Vincenti, A. Ricci, A. Malvache, R. Lopez-Martens, and F. Quéré, Attosecond Lighthouses from Plasma Mirrors, Nat. Photonics 6, 829 (2012).

[43] R. A. Ganeev, T. Witting, C. Hutchison, V. V. Strelkov, F. Frank, M. Castillejo, I. Lopez-Quintas, Z. Abdelrahman, J. W. G. Tisch, and J. P. Marangos, Comparative Studies of Resonance Enhancement of Harmonic Radiation in Indium Plasma Using Multicycle and Few-Cycle Pulses, Phys. Rev. A 88, 033838 (2013).

[44] O. Kfir et al., Generation of Bright Phase-Matched Circularly-Polarized Extreme Ultraviolet High Harmonics, Nat. Photonics 9, 99 (2014).

[45] M. Schultze et al., Controlling Dielectrics with the Electric Field of Light, Nature (London) 493, 75 (2013).

[46] M. Schultze et al., Attosecond Band-Gap Dynamics in Silicon, Science 346, 1348 (2014).

[47] S. Neppl et al., Direct Observation of Electron Propagation and Dielectric Screening on the Atomic Length Scale, Nature (London) 517, 342 (2015).

[48] T. V. Liseykina, S. Pirner, and D. Bauer, Relativistic Attosecond Electron Bunches from Laser-Illuminated Droplets, Phys. Rev. Lett. 104, 095002 (2010).

[49] T. D. Donnelly, T. Ditmire, K. Neuman, M. D. Perry, and R. W. Falcone, High-Order Harmonic Generation in Atom Clusters, Phys. Rev. Lett. 76, 2472 (1996).

[50] H. Ruf et al., Inhomogeneous High Harmonic Generation in Krypton Clusters, Phys. Rev. Lett. 110, 083902 (2013).

[51] B. Schütte, M. Arbeiter, T. Fennel, M. J. J. Vrakking, and A. Rouzée, Rare-Gas Clusters in Intense Extreme-Ultraviolet Pulses from a High-Order Harmonic Source, Phys. Rev. Lett. 112, 073003 (2014). 